\title{
The Effects of Different Quality of Exercise on Blood Pressure and Heart Rate in Healthy Female
}

\author{
Aiesha Mohammed Almutawa1, Afaf Abdullah Al-Shelash', Buthaynah Mohammed Al-Gazlan1, \\ Reema Mohammad Al-Sallali' ${ }^{1}$, Reem Abdullah Al-Marzougi ${ }^{1}$, Noorah Saleh Al-Sowayan ${ }^{2 *}$ (1) \\ ${ }^{1}$ Department of Biology, College of Science, Qassim University, Buraydah, KSA \\ ${ }^{2}$ Department of Biology, Faculty of Science, Qassim University, Buraydah, KSA \\ Email: *nsaoiean@qu.edu.sa, *knaaj1@yahoo.com
}

How to cite this paper: Almutawa, A.M., AL-Shelash, A.A., Al-Gazlan, B.M., AL-Sallali, R.M., AL-Marzougi, R.A. and Al-Sowayan, N.S. (2020) The Effects Of Different Quality of Exercise on Blood Pressure and Heart Rate in Healthy Female. Health, 12, 425-435. https://doi.org/10.4236/health.2020.124034

Received: March 28, 2020

Accepted: April 27, 2020

Published: April 30, 2020

Copyright ( 2020 by author(s) and Scientific Research Publishing Inc. This work is licensed under the Creative Commons Attribution International License (CC BY 4.0).

http://creativecommons.org/licenses/by/4.0/

(c) (i) Open Access

\begin{abstract}
Blood pressure is a serious disease that may affect a person. It also increases the risk of heart attack and stroke. Exercise may be a major reason for reducing the risk of heart disease in the future. The aim of this study is to compare the effects of different types of exercises, such as walking, cross fit, and Swedish on blood pressure and heart rate among a number of physical exercises practitioners and gymnasts. This study was conducted on pioneers of different gyms in the Qassim region after taking ethical procedures and taking the consent of the subjects' members. The subject size is 39 females between 14 38 of age. They were divided into three groups; each one had 13 members. The first experimental group practiced walking, the second practiced crossfit; lastly, the third experimental group practiced the Swedish sport. A device was used to measure the blood pressure and heart rate. The measurement was taken 5 minutes pre and post the rest period. After that the measurements were taken 15 minutes after the rest period. SPSS software was implemented to analyze the data. The significant result was that the three groups showed low blood pressure after performing various exercises while the heart rate increased in all three. This study proved that the severity of low blood pressure depends on the intensity of the exercise. The findings revealed that the highest exercise intensity is CrossFit, but also it is the highest of the decrease in blood pressure. The researchers recommend that readers should consider the importance of exercising as it is maybe a method for preventing from high blood pressure.
\end{abstract}

\section{Keywords}

Hypertension, Intensity, Resistance, Systolic Blood Pressure 


\section{Introduction}

Hypertension is one of the most spreading diseases, one of the most common causes of death in the world [1]. People with high blood pressure are more likely to have strokes and myocardial infarction than normal people because of the poor diagnosis of this disease. According to a recent World Health Organization report, relatively hypertension is considered as the main risk for cardiovascular and death [2].

And it has complications of excessive blood pressure that causes 17 million deaths each year [3] [4]. Forty-six percent of adults in the United States are affected by high blood pressure as a leading cause of death and disability due to cardiovascular disease. Despite the effectiveness of blood pressure medications, controlling blood pressure disease is not ideal [5].

Environmental factors such as stress, exercise and dietary sodium intake have frequently been linked to cardiovascular diseases. Reducing of everyday life stress, increasing the level of exercise, and reducing dietary sodium intake have been recommended as having a beneficial effect in blood pressure [6]. Symptoms of blood pressure can be prevented through non-drug treatments, such as regular physical activity and exercise. There is a relationship between exercising and low blood pressure; thus, it is recommended to lower blood pressure [4].

Physical exercise can perform several beneficial effects for health such as health boost and the ability of reducing disease [6]. The American College of Sports Medicine (ACSM) defines exercise as all activities that use a large amount of muscle. Among these exercises are walking, running, Swedish, CrossFit, and many others. Much evidence confirms that not only doing exercises continues to increase the functional ability, but it reduces the exposure to various diseases such as cardiovascular disease, blood pressure, etc. [7]. It has been shown that active people and practitioners have a lower risk of developing high blood pressure compared to others [8].

Guidelines regarding physical exercise as a factor that plays a vital role in the management of blood pressure values in hypertensive patients since this tool may collaborate with the control of blood pressure and associate with the risk factors. In this sense, systematic reviews and meta-analytic regression have proved that aerobic and resistance training programs can emerge significantly in decreasing the blood pressure values of hypertensive patients. However, the influence of other physical exercises on the hemodynamic parameters of hypertensive patients remains ambiguous [2].

Recently, multicomponent exercise programs (MCEP) have been suggested as a preferable exercise for hypertensive patients due to the design of this kind of exercise with involving various types of exercises; for instance, aerobic and resistance, the same exercise session or routine. This feature of MCEP is essential because hypertension patients avoid drawn-out physical exercise programs because of the lack of commitment [2].

Several studies that concentrate on the response of blood pressure and post-exercise heart rate have shown that doing exercises lower the blood pressure after the exercise within a short period of rest. Therefore, physical activities are 
recommended to prevent high blood pressure. However, there are some discrepancies in post-exercise blood pressure, and this depends on the length and intensity of the physical exercise [9]. Some studies have proven that doing a moderate-intense exercise for a short period would be sufficient to trigger blood pressure response [10]. On the other hand, others indicated that doing a low to moderate exercise did not cause any changes to the heart rate levels and blood pressure after the exercise. Thus, this proves that the relationship of neurovascular circulation response during the exercise is directly related to the intensity of the exercise [9]. The studies are different regarding the experimental protocol in terms of the participants in the study, the duration, and the intensity of the exercise and its method.

Although the antihypertensive mechanisms of exercise are not completely understood. Yet enormous mechanisms are projected to be involved in the reduction of blood pressure while exercising such as decreasing the levels of angiotensin 11 [6]. Only a few studies have been designed to study the effect of exercise on blood pressure levels. Moreover, these few studies are based on experiments performed with low participants sizes and Using non blinded method to blood pressure measurement [2].

In recent years, there is an increase interest of the effects of short-terms exercise training cardiovascular system [6]. The present study aimed to compare the effects of three different types of exercises, such as Swedish, CrossFit and Walking, on blood pressure and heart rate to Show the benefit of these exercises. To define this the researchers used Heart Rate (HR) which means the speed of the heartbeat, which is measured by the number of beats (pulses) of the heart per minute [11]; and blood pressure (BP) which refers to the rate of blood pressure in an artery. When the heart muscle contracts and blood is injected, it is called systolic blood pressure. And when the heart muscle relaxes, it is called diastolic blood pressure [12].

\section{Material and Methods}

\subsection{Subjects}

In this study, the researchers have conducted a study on 39 healthy female participants. These participants are between the ages 14 and 38 years old. The researchers divided the subjects into 3 groups of 13. They all gym goers and do regular physical activities.

To ensure the right outcomes, all participants do not have history of cardiovascular disease or any other illnesses. Furthermore, they do not take any drugs (1). The researchers have excluded certain subjects who underwent menstruation, and the ones who had systolic blood pressure (SYS) $>144 \mathrm{~mm} \mathrm{Hg}$ diastolic blood pressure (DIA) $>106 \mathrm{mmHg}$ body mass index (BMI) $>38.90 \mathrm{Kg} / \mathrm{m}^{2}, \mathrm{HR}>$ 114. Thus, the experiment was introduced to all participants and all gave their written consent to participate.

\subsection{Procedures}

The researchers have measured the $\mathrm{HR}, \mathrm{BP}, \mathrm{BMI}$, weight and height, before 
starting the experiment. In this study, the subjects were divided into three different groups. Each group was assigned to do a particular exercise. The exercises that assigned were walking, CrossFit, and Swedish.

To explain this, the walking exercise which moves the whole body or continuous rotation (mono-structural) movements [13]. CrossFit exercise is a new strength and an adaptable program with a short but intense daily workouts. These workouts include strength and resistance training with elastic resistance bands [14]. Swedish exercise (Core) is Known for strengthen the deep muscles of the human body, such as the spinal muscles, abdominal muscles, hip muscles, and the pelvic muscles [15].

Each exercise was performed for 40 minutes. The rate of BP and HR were measured pre-and-post-exercise. All measurements were recorded at $4-6$ p.m. The researchers urged all participants to avoid any strenuous physical activities 48 hours prior to the assigned exercise, and the last meal should be eaten two hours before the exercise time [16].

The blood pressure and heart rate measurement is taken from the participants in the sitting position before exercise. After that, BP and HR were measured 5 minutes after the exercise and then 15 minutes at rest period; total session is 65 minutes. The study was carried out on two separate visits. The second visit is a repetition of the first one. BP and HR were measured using Blood Pressure Monitor Model BP5100 (OMRON).

\subsection{Statistical Analysis}

In this study, SPSS v. 23 software has been used to for variance analysis (ANOVA) in repetitive measures ( 3 exercises $\times 3$ timepoint). Each exercise has 13 subjects. Descriptive statistic tests and Bonferroni post hoc tests were used in order to analyze the data. All data analysis implemented at Probability Value $\mathrm{p} \leq 0.05$ level.

\section{Results}

There was no adverse events occurred during the experiment. Volunteers did not report any changes in the core values of body weight, BMI, height and their ages during the study. The overall average values of participants according to the average of SYS, DIA and HR are shown in (see Table 1). BP significantly decreased after each type of exercise. Also there was a significant difference among crossFit exercise (CE), walk exercise (WE) and swedish exercise (SE).

SYS significantly decreased after $\mathrm{CE}, \mathrm{WE}$ and $\mathrm{SE}$ in comparison to the rest period values before performing exercise during the two times 5 and $15 \mathrm{mi}$ nutes. However, WE increased SYS values after 5 minutes of the exercise, then it decreased after 15 minutes (Figure 1).

This trail obviously proved that CE have the most positive effect on decreasing SYS; pre-exercise $(127.69 \pm 11.16)$ post-exercise $(107.07 \pm 13.85)$ in (Table 2$)$. Also DIA decreased after all three types of exercises during the two time points except for WE; the DIA increased after 5 minutes, then it decreased after $15 \mathrm{mi}-$ nutes (Figure 2). Furthermore, CE has the most effect on decreasing DIA: 
pre-exercise $(87.00 \pm 11.59)$ post-exercise $(78.15 \pm 12.12)$ (see Table 3$)$.

The average of age, weight, and height were quantitively continuum variables that researchers have measured during the trial (Table 1 ). In the case of SYS, DIA and $\mathrm{HR}$ variables were quantitively discrete variables that researchers have compared in Table 2 \& Table 3 and also in Figures 1-3 between pre-exercise and post exercise for each type of exercise. The intensity of exercise type and time were independent variables that have effect on SYS, DIA and HR that were dependent variables.

Table 1. Results of Physical and cardiovascular characteristic of the participants.

\begin{tabular}{cccc}
\hline Variables & Mean \pm SD & Variables & Mean \pm SD \\
\hline Age (year) & $25.12 \pm 5.25$ & SYS $(\mathrm{mmHg})$ & $121.12 \pm 11.50$ \\
Weight $(\mathrm{Kg})$ & $63.87 \pm 12.44$ & DIA $(\mathrm{mmHg})$ & $81.48 \pm 10.00$ \\
Height $(\mathrm{Cm})$ & $156.38 \pm 10.67$ & HR $(\mathrm{bpm})$ & $85.28 \pm 14.63$ \\
$\left(\mathrm{Kg} / \mathrm{m}^{2}\right)$ & $25.70 \pm 5.02$ & & \\
\hline
\end{tabular}

Table 2. Comparison of (SYS) Per-and Post-exercise WE: Walking exercise; CE: CrossFit exercises; SE: Swedish exercise a significant difference VS res analysis implemented at $\mathrm{p} \leq$ 0.05 level.

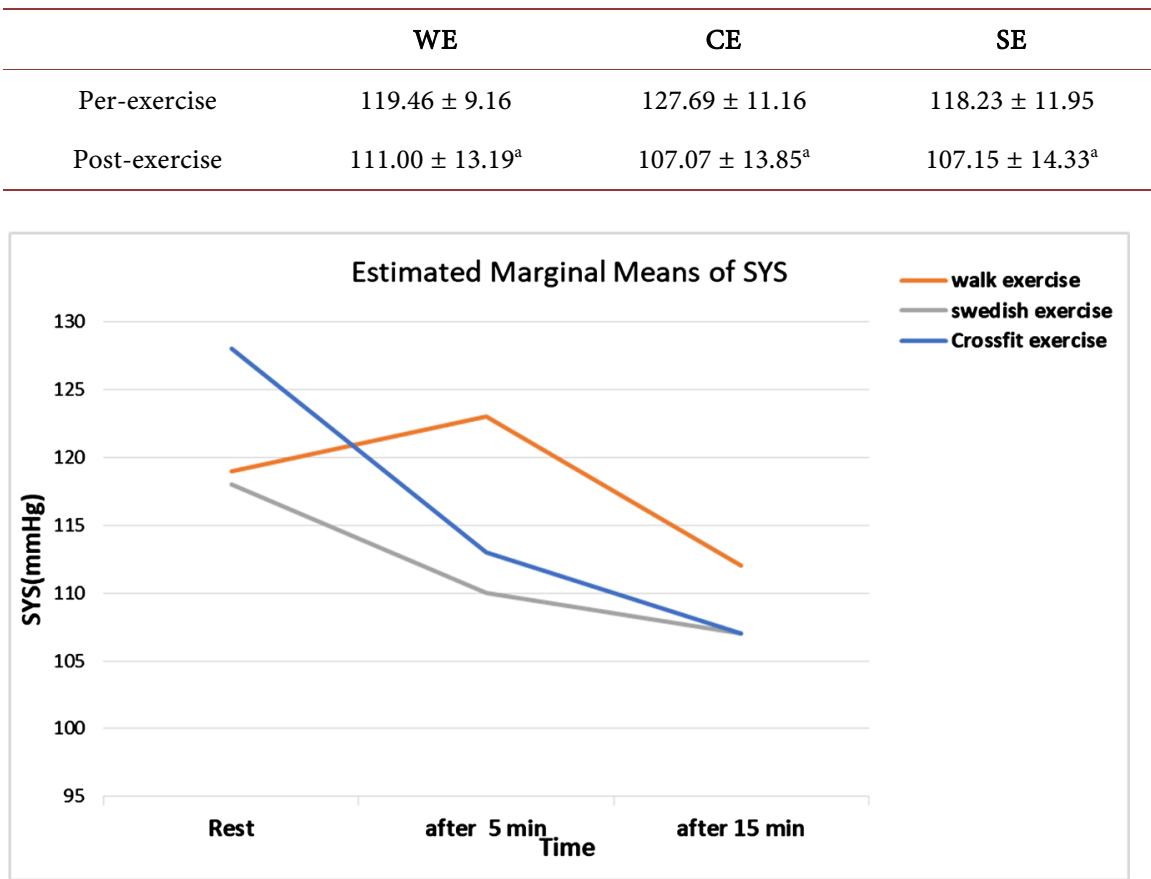

Figure 1. Changes in systolic blood pressure.

Table 3. Comparison of (DIA) Per-and Post-exercise WE: Walking exercise; CE: CrossFit exercises; SE: Swedish exercises ${ }^{a}$ significant difference VS rest analysis implemented at $\mathrm{p}$ $\leq 0.05$ level.

\begin{tabular}{cccc}
\hline & WE & CE & SE \\
\hline Per-exercise & $77.69 \pm 8.46$ & $87.00 \pm 11.59$ & $80.38 \pm 7.52$ \\
Post-exercise & $72.84 \pm 9.30^{\mathrm{a}}$ & $78.15 \pm 12.12^{\mathrm{a}}$ & $76.61 \pm 7.21^{\mathrm{a}}$ \\
\hline
\end{tabular}




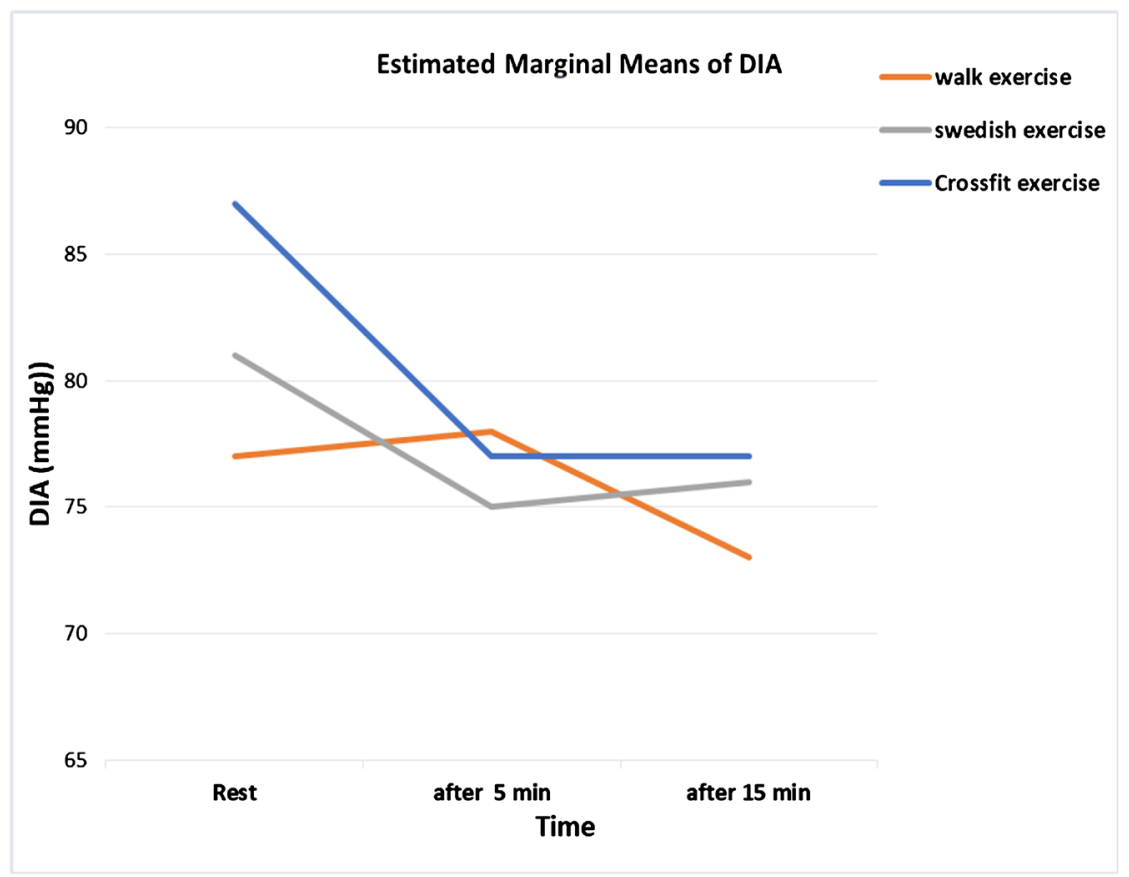

Figure 2. Changes in diastolic blood pressure.

Table 4. Comparison (HR) Per- and Post-exercise WE: Walking exercise; CE: CrossFit exercises; SE: Swedish exercises a significant difference VS rest analysis implemented at $\mathrm{p}$ $\leq 0.05$ level.

\begin{tabular}{cccc}
\hline & WE & CE & SE \\
\hline Per-exercise & $92.07 \pm 12.64$ & $87.38 \pm 15.48$ & $79.92 \pm 16.39$ \\
Post-exercise & $97.15 \pm 12.96^{\mathrm{a}}$ & $98.69 \pm 10.80^{\mathrm{a}}$ & $79.92 \pm 10.91$ \\
\hline
\end{tabular}

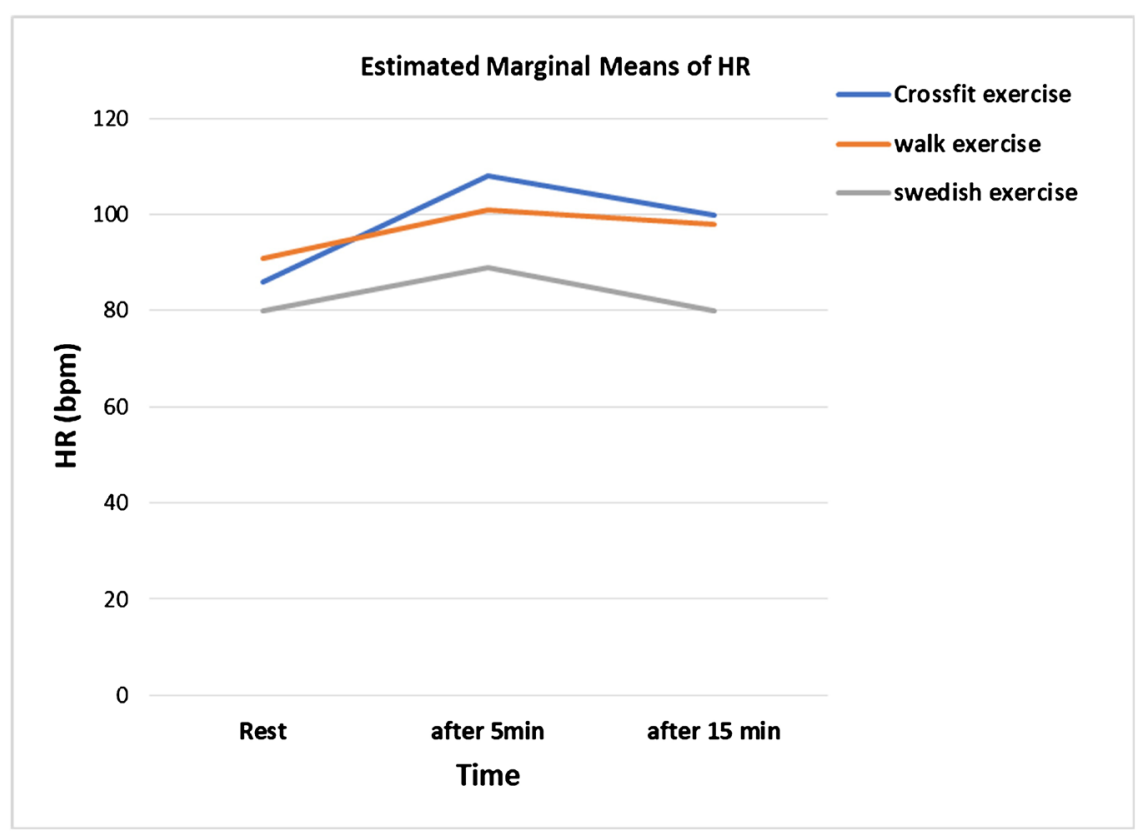

Figure 3. Changes in heart rate. 


\section{Discussion}

In the current study, it proved that crossfit, swedish, walking exercises all can reduce blood pressure after exercise, but the degree of reduction varies depending on the intensity of the exercise. The intensity of the exercise is measured by the Rating of Perceived Exercise (RPE) that was developed by Borg Rating which is a quantitative measure used to measure physical activity effort to assess training intensity [17]. RPE is rated $6-20$ as the scale of 6 no exertion at all, low intensity is between $7-11$, medium intensity is $12-14$, and high intensity is 15 20 [18]. All three types of exercises the study is dependent on increased the heart rate. There is a gradual increase depending on the intensity of the exercise in this study. The highest of the decrease in blood pressure and the increase in heart rate was recorded for crossefit exercise because it is the highest intensity exercise.

HR increased significantly after CE and WE increased after 15 minutes compared to the rest period (Figure 3). CE increased HR more than WE: pre-exercise $(87.38 \pm 15.48)$ post-exercise $(98.69 \pm 10.80)$ (see Table 4$)$. There were no significant differences observed between pre-exercise and post-exercise values in SE of HR. Researchers expect that HR values decrease if participants were given a longer rest time after the exercise. And that can be proven from the differences among the records after 5 and 15 minutes.

Increasing exercise intensity is to accomplish a certain amount of total exercise in a short period of time. Doctors recommend carrying out high-intensity exercise in order to make better use of the exercise [19]. In [20] explained after high-intensity exercise, the blood pressure drops as SYS drops significantly after $90 \mathrm{~min}$ of exercise by $75 \% \mathrm{VO}_{2}$ peak. In contrast, the DIA drops after $30 \mathrm{~min}$ of high-intensity exercise by $50 \% \mathrm{VO}_{2}$ peak.

Previous studies have shown the effect of exercise intensity on hemodynamic regulatory and neurological responses during different exercises [21] [22]. Where [9] study explained The intensity of exercise varied from $30 \%$ to $80 \%$ of $\mathrm{VO}_{2}$ peak in young people with normal blood pressure did not influence on the size of hypotension after exercise. However, unlike exercises at $50 \%$ and $80 \%$ of $\mathrm{VO}_{2}$ peak, exercises. However, unlike exercises at $50 \%$ and $80 \%$ of $\mathrm{VO}_{2}$ peak, exercises at $30 \%$ of $\mathrm{VO}_{2}$ peak may increase heart rate levels post-exercise. As [9] explained BP responses depending on the intensity of the exercise. [23] noted that extreme exercises cause hypotension after exercise in normal humans.

In another study, the increase of aerobic activities is important to prevent high blood pressure as exercising has lowered blood pressure in people with high blood pressure and normal blood pressure [24]. [10] mentioned that extreme exercises in both high intensity (HI) and low intensity (LI) training reduce SYS significantly and to certain extent whereas there was no decrease in SYS after moderate intense exercise. Cornelissen [10] noted that high intensity had a remarkable effect on human during rest, exercise, and post-exercise.

On other hand, [25] said that exercises with a low intensity f $40 \%-50 \% \mathrm{VO}_{2}$ maximum seems as effective as exercising in medium intensity $70 \% \mathrm{VO}_{2}$ maxi- 
mum in order to reduce BP 7 hours post-exercise. A study by [26] also demonstrated that moderate exercises reduced BP and HR in middle-aged women. The study proved that change in troponin of the heart muscle is caused by stressful exercise. By warming-up before the exercise and taking vitamin E can prevent heart damage [27].

There is a disagreement on the issue of the impact of the exercising time on pressure rate (PR)and HR. [28] concluded that there was a hypotensive response at 15, 30 and 45 minutes of the exercise. It also showed that 15 minutes of the exercise caused a decrease in blood pressure similar to post-exercise as $45 \mathrm{mi}$ nutes of exercise. [28] stated from MacDonald JR (1991) \& Pescatello LS (1991) that the moderate intensity exercise for a short period 15 minutes is sufficient enough to stimulate a blood pressure response reduction.

This contrasts with the study by [29]. It stated that by increasing the volume of blood pressure is linked to the increase of the exercise duration. [23] reported that 15 minutes of exercise did not stimulate hypotension after exercising.

A study by [16] showed SYS decreased significantly after resistance exercise R15, R30, R45, R60 minutes. However, the DIA which did not change post-exercise at R45 \& R60 minutes. It is important to mention that the condition of post-exercise hypotension (PEH) should be accurately described.

\section{Conclusion}

Overall, the present study indicates that there are a beneficial effect of doing exercises, such as walking, Swedish and crossfit; especially, exercises with high intensity like crossfit in order to decrease blood pressure in healthy people. Researchers give advice to patients with hypertension to do exercises especially with high intensity to protect themselves from strokes, myocardial infarction and other risks.

\section{Acknowledgements}

We would like to thank everyone who participated in this study. A special gratitude to our professor Noorah AL, Sowyan for supporting us in order to accomplish this research. Also we are grateful for the gym management for allowing us to conduct our study there.

\section{Conflicts of Interest}

The authors declare no conflicts of interest regarding the publication of this paper.

\section{References}

[1] Bell, T.P., Mcintyre, K.A. and Hadley, R. (2014) Effect of Long-Term Physical Exercise on Blood Pressure in African Americans. International Journal of Exercise Science, 7, 3. https://digitalcommons.wku.edu/ijes/vol7/iss3/3

[2] Coelho-Júnior, H.J., Asano, R.Y., Gonçalvez, I.D.O., Brietzke, C., Pires, F.O., Aguiar, S.D.S., Rodrigues, B., et al. (2018) Multicomponent Exercise Decreases Blood Pressure, Heart Rate and Double Product in Normotensive and Hypertensive 
Older Patients with High Blood Pressure. Archivos de Cardiología de México, 88, 413-422. https://doi.org/10.1016/j.acmx.2018.01.001

[3] MacDonald, H.V. and Pescatello, L.S. (2019) Exercise and Blood Pressure Control in Hypertension. In: Cardiorespiratory Fitness in Cardiometabolic Diseases, Springer, Cham, 137-168. https://doi.org/10.1007/978-3-030-04816-7 8

[4] Heitmann, K.A., Dalen, H., Holmen Gundersen, G., Ingvaldsen, R.P. and Welde, B. (2019) Intra-Arterial Blood Pressure Traits during and after Heavy Resistance Exercise in Healthy Males. Translational Sports Medicine, 2, 325-333.

https://doi.org/10.1002/tsm2.102

[5] Persell, S.D., Peprah, Y.A., Lipiszko, D., et al. (2020) Effect of Home Blood Pressure Monitoring via a Smartphone Hypertension Coaching Application or Tracking Application on Adults with Uncontrolled Hypertension: A Randomized Clinical Trial. JAMA Network Open, 3, e200255.

https://doi.org/10.1001/jamanetworkopen.2020.0255

[6] Jakovljevic, B., Plecevic, S., Petkovic, A., Turnic, T.N., Milosavljevic, I., Radoman, K. and Srejovic, I. (2019) Is 3 Weeks of Exercise Enough to Change Blood Pressure and Cardiac Redox State in Hypertensive Rats? Serbian Journal of Experimental and Clinical Research, 20, 319-326. https://doi.org/10.1515/sjecr-2017-0049

[7] Desai, B. and Desai, D. (2020) To Compare the Effect of Specific Yoga and Aerobic Exercise Program on Vital Parameters in Young Adult Females. International Journal of Current Research and Review, 12, 1. https://doi.org/10.31782/IJCRR.2020.12021

[8] Cardoso, J.R., Gomes, C., et al. (2010) Acute and Chronic Effects of Aerobic and Resistance Exercise on Ambulatory Blood Pressure. Clinics, 65, 317-325. https://doi.org/10.1590/S1807-59322010000300013

[9] Forjaz, C.L.M., Matsudaira, Y., Rodrigues, F.B., Nunes, N. and Negrão, C.E. (1998) Post-Exercise Changes in Blood Pressure, Heart Rate and Rate Pressure Product at Different Exercise Intensities in Normotensive Humans. Brazilian Journal of Medical and Biological Research, 31, 1247-1255. https://doi.org/10.1590/S0100-879X1998001000003

[10] Cornelissen, V.A., Verheyden, B., Aubert, A.E. and Fagard, R.H. (2010) Effects of Aerobic Training Intensity on Resting, Exercise and Post-Exercise Blood Pressure, Heart Rate and Heart-Rate Variability. Journal of Human Hypertension, 24, 175-182. https://doi.org/10.1038/jhh.2009.51

[11] Spodick, D.H., Raju, P., Bishop, R.L. and Rifkin, R.D. (1992) Operational Definition of Normal Sinus Heart Rate. The American Journal of Cardiology, 69, 1245-1246. https://doi.org/10.1016/0002-9149(92)90947-W

[12] American Heart Association (2017) What Is High Blood Pressure? South Carolina State Documents Depository.

[13] Butcher, S.J., Judd, T.B., Benko, C.R., Horvey, K.J. and Pshyk, A.D. (2015) Relative Intensity of Two Types of Crossfit Exercise: Acute Circuit and High-Intensity Interval Exercise. Journal of Fitness Research, 4, 3-15.

[14] Joondeph, S.A. and Joondeph, B.C. (2013) Retinal Detachment Due to Crossfit Training Injury. Case Reports in Ophthalmological Medicine, 2013, Article ID: 189837. https://doi.org/10.1155/2013/189837

[15] Yoon, S.-D., Sung, D.-H. and Park, D. (2015) The Effect of Active Core Exercise on Fitness and Foot Pressure in Taekwondo Club Students. Journal of Physical Therapy Science, 27, 509-511. http://creativecommons.org

https://doi.org/10.1589/jpts.27.509 
[16] Mohebbi, H., Rohani, H. and Ghiasi, A. (2016) Effect of Involved Muscle Mass in Resistance Exercise on Post Exercise Blood Pressure and Pressure Product. Apunts Medicina de P Esport, 51, 123-129. http://www.apunts/org https://doi.org/10.1016/j.apunts.2016.05.002

[17] Dawes, H.N., Barker, K.L., Cockburn, J., Roach, N., Scott, O. and Wade, D. (2005) Borg's Rating of Perceived Exertion Scales: Do the Verbal Anchors Mean the Same for Different Clinical Groups? Archives of Physical Medicine and Rehabilitation, 86, 912-916. https://doi.org/10.1016/j.apmr.2004.10.043

[18] Chen, Y.L., Chen, C.C., Hsia, P.Y. and Lin, S.K. (2013) Relationships of Borg's RPE 6-20 Scale and Heart Rate in Dynamic and Static Exercises among a Sample of Young Taiwanese Men. Perceptual and Motor Skills, 117, 971-982. https://doi.org/10.2466/03.08.PMS.117x32z6

[19] Meltzer, D.O. and Jena, A.B. (2010) The Economics of Intense Exercise. Journal of Health Economics, 29, 347-352. https://doi.org/10.1016/j.jhealeco.2010.03.005

[20] Forjaz, C.L.M., Cardoso, C.G., Rezk, C.C., Santaella, D.F. and Tinucci, T. (2004) Postexercise Hypotension and Hemodynamics: The Role of Exercise Intensity. Journal of Sports Medicine and Physical Fitness, 44, 54-62.

[21] Leuenberger, U., Sinoway, L., Gubin, S., Gaul, L., Davis, D. and Zelis, R. (1993) Effects of Exercise Intensity and Duration on Norepinephrine Spillover and Clearance in Humans. Journal of Applied Physiology, 75, 668-674. https://doi.org/10.1152/jappl.1993.75.2.668

[22] Saito, M., Tsukanaka, A., Yanagihara, D. and Mano, T. (1993) Muscle Sympathetic Nerve Responses to Graded Leg Cycling. Journal of Applied Physiology, 75, 663-557. https://doi.org/10.1152/jappl.1993.75.2.663

[23] Piepoli, M., Isea, J.E., Pannarale, G., Adamopoulos, S., Sleight, P. and Coats, A.J.S. (1994) Load Dependence of Changes in Forum and Peripheral Vascular Resistance after Acute Leg Exercise in Man. Journal of Physiology, 478, 357-362.

http://physoc.onlinelibrary.wiley.com https://doi.org/10.1113/jphysiol.1994.sp020256

[24] Whelton, S.P., Chin, A., Xin, X. and He, J. (2002) Effect of Aerobic Exercise on Blood Pressure: A Meta-Analysis of Randomized Controlled Trials. Annals of Internal Medicine, 136, 493-503. https://doi.org/10.7326/0003-4819-136-7-200204020-00006

[25] Kokkinos, P.F., et al. (1995) Effects of Regular Exercise on Blood Pressure and Left Ventricular Hypertrophy in African-American Men with Severe Hypertension. The New England Journal of Medicine, 333, 1462-1467. https://doi.org/10.1056/NEJM199511303332204

[26] Imamoglu, O., Akyol, P. and Satici, A. (2017) The Effect of Aerobic Exercise and Weight-Lifting plus Aerobic Exercise on Blood Pressure and Blood Parameters in Sedentary Females. European Journal of Physical Education and Sport Science, 3, 1-8.

[27] Al-Sowyan, N.S. (2010) Effect of Exercise and Vitamin E on Cardiac Troponin Alterations in Myocardium and Serum of Rats after Stressful Intense Exercise. International Journal of Zoological Research, 6, 24-29.

http://www.academicjournals.com https://doi.org/10.3923/ijzr.2010.24.29

[28] MacDonald, J.R., MacDougall, J.D. and Hogben, C.D. (2000) The Effects of Exercise Duration on Post-Exercise Hypotension. Journal of Human Hypertension, 14, 125-129. http://www.nature.com/jhh 
https://doi.org/10.1038/sj.jhh.1000953

[29] Bennett, T., Wilcox, R.G. and MacDonald, I.A. (1984) Post-Exercise Reduction of Blood Pressure in Hypertensive Men Is Not Due to Acute Impairment of Baroreflex Function. Clinical Science, 67, 97-103. http://portlandpress.com https://doi.org/10.1042/cs0670097 